\title{
Bubble size analysis generated on the submerged orifice, depending on the gas supply mode
}

\author{
Sergey Goldaev ${ }^{1}$, Sergey Basalaev ${ }^{2,}$, and Kirill Afanasyev ${ }^{3}$ \\ ${ }^{1}$ National Research Tomsk Polytechnic University, 634050 Tomsk, Russia \\ ${ }^{2}$ Tomsk State University, 634050 Tomsk, Russia \\ ${ }^{3}$ Transneft Central Siberia, 634050 Tomsk, Russia
}

\begin{abstract}
A numerical analysis of an algebraic equation of the fifth degree describing the achievement of the diameter of a gas bubble at an aperture is made, the results obtained earlier for limiting cases by simplifying the initial equation and solving it by an analytical method are refined and generalized. With the help of the ratio of the Froude criterion to the Weber criterion, the boundaries of realization of the static, transient and dynamic regimes are established. Approximating formulas are proposed for these regimes, which, with an allowable error in the performance of applied calculations, make it possible to find the value of the detachment diameter of the bubble.
\end{abstract}

\section{Introduction}

Process of formation of bubbles of gas on opening cut at its expiration in liquid accompanies aeration processing [1], when heating water with devices of submersible burning [2], stage of cooling of combustion products before filling of covers of buoyancy [3].

The surface area of gazo-liquid structure having impact on efficiency of the considered processes represents total surface of bubbles of the different sizes [2].

It is known that with reduction of diameter of bubbles, the size of phase boundary at identical gas content increases in bubbling layer. Therefore decrease in the sizes of bubbles is one of ways of increase of efficiency of processes of gas-liquid contact [2].

Dynamics of education and separation of bubbles at the expiration of gas in liquid through small opening is described by diameter of $d_{0}$ in work [4]. Eighteen publications in which the question by determination of detachable diameter of bubble of $D$ was considered are mentioned in its survey part, is noted that there is no uniform model of this process. Some semi-empirical techniques are given in the monograph [2] according to $D$ which with different accuracy describe the considered process, however results from work [4] are not mentioned.

\footnotetext{
* Corresponding author: tarm@,niipmm.tsu.ru
} 
Fig. 1 shows the scheme for the formation of a gas bubble of diameter $D$ through a small hole $d_{0}$ located at a depth $h$, a gas flow with velocity $v_{0}$.

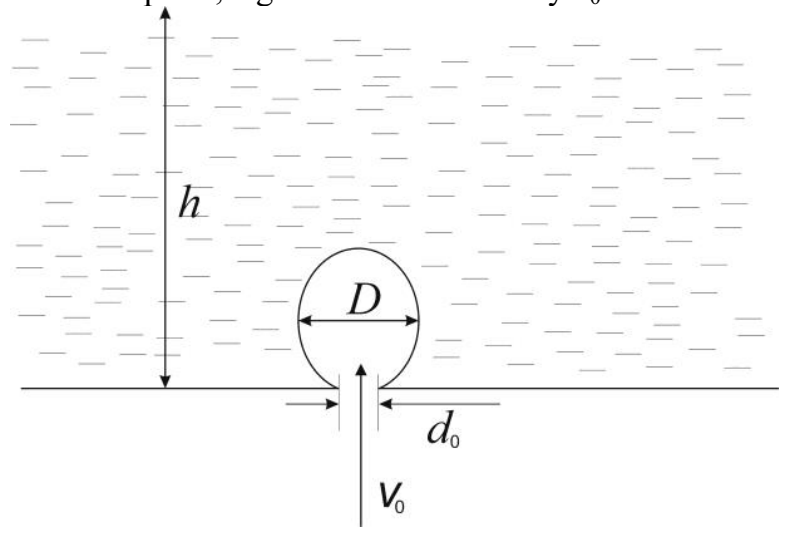

Fig. 1. Scheme of formation of the gas.

The purpose of the real work consists in quantitative analysis of the size of detachable diameter of bubble depending on parameters of the considered process by the numerical solution of the equation proposed in [4] and receiving the approximating expressions allowing to carry out evaluation calculations at preliminary stage of creation of bubblers.

\section{Analysis results calculation bubble diameter}

In work [4] on the basis of use of equilibrium condition of the gas bubble which is at the time of separation across small hole under the influence of four forces: lifting, surface tension, hydrodynamic pressure of gas and inertial force of liquid, the equation for calculation of detachable diameter of gas bubble is received.

$$
L^{5}+\left(1.5 K_{g} \cdot \mathrm{Fr}-6 \cdot \mathrm{We}\right) L^{2}=4 \cdot K_{w} \cdot \mathrm{Fr},
$$

where $\mathrm{We}=\sigma /\left[\left(\rho_{w}-\rho_{g}\right) g d_{0}^{2}\right] \quad$ Weber's number; $\mathrm{Fr}=v_{0}^{2} / g d_{0} \quad$ Froude number; dimensionless parameters $K_{g}=\rho_{g} /\left(\rho_{w}-\rho_{g}\right) ; K_{w}=\rho_{w} /\left(\rho_{w}-\rho_{g}\right) ; \quad \rho_{g}, \rho_{w}-$ gas densities and liquids; $\sigma$ coefficient of surface tension; $g$ acceleration due to gravity; $v_{0}$ gas exhaust speed in liquid; $D, d_{0}$ bubble and hole diameters.

Analysis of the values of the ratio $\delta_{F W}=\left(K_{g} / 4\right) \cdot \mathrm{Fr} / \mathrm{We}$ allows us to clarify the boundaries of the implementation of a particular regime.

Quantitative results were obtained in [4] for limiting cases when it was possible to simplify the initial equation (1) and solve it analytically. Specific values of the criteria We and Fr for the respective modes are not given.

In this paper we present the results of a detailed analysis of the effect of the components under consideration on $L$.

The equation (1) has been solved by method of bisektion [5]. During calculations $d_{0}=1 \ldots 6 \mathrm{~mm}, v_{0}=0.25 \ldots 20 \mathrm{~m} / \mathrm{s}$ which corresponded to the experimental data [4] varied.

A comparison of the calculated data with the experimental data from [6] showed a satisfactory agreement. 
Taking into account the possible use of bubbling devices in high-pressure plants or gas generators to fill hard and soft pontoons located at a depth of several hundred meters [3], the influence of this factor, which affects the density of the gas filling the bubble, was estimated.

To calculate the bubble diameters in the case of the action of a large hydrostatic pressure, an approximate formula

$$
L_{p h}=k_{h}\left(4 \cdot K_{w} \cdot \mathrm{Fr}\right)^{0.2},
$$

where $k_{h}=\left\{\begin{array}{c}1, h<250 ; \\ \mathrm{We}+\left(0.005 h / h_{1}\right)^{-0.7} .\end{array}\right.$

Calculations have shown that with depth $h=500 \mathrm{~m}$ value $L_{p h}=5.62$, which for $5 \%$ exceeds diameter of the bubble found the numerical solution of the equation (1), and at $h=1000 \mathrm{M}-L_{p h}=4.49$, and it is $2 \%$ more than value of $L$.

Simulation of the static regime of bubble formation was accomplished by setting low rates of gas outflow into water.

It is established that at $d_{0}=1 \mathrm{~mm}$ and exhaust speeds of $v_{0}<0.75 \mathrm{~m} / \mathrm{s}$, are carried out inequality $\operatorname{Fr}<<K_{L 3}$, and the error of calculation for formula (2) is in tolerance limits. Therefore it is possible to say that the static mode is implemented. However for diameters of $d_{0}=3 \ldots 5 \mathrm{~mm}$ and small exhaust speeds the mentioned inequality is not carried out that leads to big error of calculation of $L$ for formula (2).

In order to generalize the calculated data for the static and transient regimes by the method of selected points [4], an interpolation formula was obtained containing the Froude and Weber criteria:

$$
L_{a}=\left(1.296+0.493 \mathrm{Fr}^{0.318}\right) k_{c}
$$

where $k_{c}=\left\{\begin{array}{cc}1 /(1+0.25 \mathrm{We}), & \mathrm{We}<0.85 \\ \mathrm{We} /(1+0.65 \mathrm{We}), & \mathrm{We}>0.85\end{array}\right.$.

Table 1 shows the results of the calculation of the dimensionless diameter of the $L_{\mathrm{a}}$ bubble according to this formula, and also the relative error in comparison with the values of $L$.

Table 1. Diameter of the bubble at different flow rates and hole diameters.

\begin{tabular}{|c|c|c|c|c|c|}
\hline \multirow{2}{*}{$\begin{array}{c}v_{0}, \\
\mathrm{~m} / \mathrm{s}\end{array}$} & 0.2 & 0.4 & 0.6 & 0.8 & 1.0 \\
\cline { 2 - 6 } & \multicolumn{5}{|c|}{$d_{0}=0.003 \mathrm{~m} ; \mathrm{We}=0.85$} \\
\hline $\mathrm{Fr}$ & 1.36 & 5.44 & 12.2 & 21.8 & 34 \\
\hline$\delta_{F W} \cdot 10^{3}$ & 0.55 & 2.2 & 4.9 & 8 & 14 \\
\hline$L$ & 1.88 & 2.14 & 2.39 & 2.61 & 2.81 \\
\hline$L_{a}$ & 1.52 & 1.77 & 1.98 & 2.16 & 3.07 \\
\hline & \multicolumn{5}{|c|}{$d_{0}=0.005 \mathrm{~m} ; \mathrm{We}=0.31$} \\
\hline $\mathrm{Fr}$ & 0.8 & 3.26 & 7.34 & 13.04 & 20.4 \\
\hline$\delta_{F W} \cdot 10^{3}$ & 0.9 & 3.7 & 8.2 & 15 & 22.9 \\
\hline$L$ & 1.49 & 1.80 & 2.06 & 2.28 & 2.47 \\
\hline$L_{a}$ & 1.64 & 4.20 & 4.94 & 5.54 & 6.06 \\
\hline
\end{tabular}


It is established that the proposed approximation formula describes, with an error not exceeding $10 \%$, the results of solving equation (1) in the static and transition regions.

Since the volume flow of gas through the hole, the diameter of the bubble and the frequency of its formation are related by the relation [2]

$$
Q_{0}=f \pi D^{3} / 6,
$$

where $f$ the frequency of forming of gas bubble in liquid layer calculated on formula

$$
f=1.5\left(v_{0} / D\right)\left(d_{0} / D\right)^{2},
$$

Thus, the calculation of the diameter of the bubble with greater accuracy makes it possible to reasonably choose the parameters of the bubbler.

\section{Conclusion}

By numerical solution of the algebraic equation of the fifth degree describing the achievement of the diameter of the gas bubble on the hole, the results obtained earlier for limiting cases by simplifying the initial equation and solving it by an analytical method are refined and generalized. It is established that at values of parameter $\delta_{F \mathrm{~W}} \approx 10^{-4} \ldots 10^{-3}$ the static mode takes place, at $\delta_{F \mathrm{~W}} \approx 10^{-3} \ldots 7 \cdot 10^{-2}$ transitional, at $\delta_{F \mathrm{~W}}>7 \cdot 10^{-2}$ the dynamic. Approximating formulas for the listed modes which with admissible error of performance of applied calculations allow to find value of detachable diameter of bubble are offered.

Research is funded by Russian Science Foundation grant (project No 15-19-10014).

\section{References}

1. S.S. Kutateladze, M.A. Styrikovich, Gidrodinamik of gas-liquid systems (Energy, Moscow, 1976) [in Russian]

2. A.N. Alabovsky, P.G. Udyma, Devices of submersible burning (Publishing house of MEI, Moscow, 1994) [in Russian]

3. V.D. Barsukov, S.V. Goldaev, N.P. Min'kova S.A. Basalaev, Underwater burning solid fuels dibasic (Publishing house of Tom., Tomsk, 2016) [in Russian]

4. A.A. Voloshko, A.V. Vurgaft, V. N. Frolov, J. Engin. Phys. Thermophys. 35 (2), 213 (1978)

5. L.I. Turchak, P.V. Plotnikov. Basics of numerical methods (Fizmatlit, Moscow, 2005) [in Russian]

6. V.P. Myasnikov, A.P. Mitronov, N.A. Kochergin, V.V. Dil'man, Dokl. Academy Sci. of the USSR, 269 (4), 827 (1983) [in Russian] 